\title{
Ex vivo rabbit and human corneas as models for bacterial and fungal keratitis
}

\author{
Abigail Pinnock ${ }^{1}$ - Nagaveni Shivshetty ${ }^{2} \cdot$ Sanhita Roy $^{2} \cdot$ Stephen $_{\text {Rimmer }}{ }^{3}$. \\ Ian Douglas ${ }^{1} \cdot$ Sheila MacNeil ${ }^{1,4} \cdot$ Prashant Garg $^{2}$
}

Received: 8 August 2016 /Revised: 23 October 2016 / Accepted: 31 October 2016/Published online: 14 November 2016

(C) The Author(s) 2016. This article is published with open access at Springerlink.com

\begin{abstract}
Purpose In the study of microbial keratitis, in vivo animal models often require a large number of animals, and in vitro monolayer cell culture does not maintain the threedimensional structure of the tissues or cell-to-cell communication of in vivo models. Here, we propose reproducible ex vivo models of single- and dual-infection keratitis as an alternative to in vivo and in vitro models.

Methods Excised rabbit and human corneoscleral rims maintained in organ culture were infected using $10^{8}$ cells of Staphylococcus aureus, Pseudomonas aeruginosa, Candida albicans or Fusarium solani. The infection was introduced by wounding with a scalpel and exposing corneas to the microbial suspension or by intrastromal injection. Post-inoculation, corneas were maintained for 24 and $48 \mathrm{~h}$ at $37^{\circ} \mathrm{C}$. After incubation, corneas were either homogenised to determine colony-forming units (CFU)/cornea or processed for histological examination using routine staining methods. Single- and mixed-species infections were compared.

Results We observed a significant increase in CFU after $48 \mathrm{~h}$ compared to $24 \mathrm{~h}$ with $S$. aureus and P. aeruginosa. However, no such increase was observed in corneas infected with C. albicans or $F$. solani. The injection method yielded an approximately two- to 100 -fold increase $(p<0.05)$ in the
\end{abstract}

Sheila MacNeil

s.macneil@sheffield.ac.uk

University of Sheffield, Sheffield S10 2TA, UK

LV Prasad Eye Institute, Banjara Hills, Hyderabad 500034, India

University of Bradford, Bradford BD7 1DP, UK

4 The Kroto Research Institute, North Campus, University of Sheffield, Broad Lane, Sheffield S3 7HQ, UK majority of organisms from infected corneas. Histology of the scalpel-wounded and injection models indicated extensive infiltration of $P$. aeruginosa throughout the entire cornea, with less infiltration observed for $S$. aureus, C. albicans and F. solani. The models also supported dual infections.

Conclusions Both scalpel wounding and injection methods are suitable for inducing infection of ex vivo rabbit and human cornea models. These simple and reproducible models will be useful as an alternative to in vitro and in vivo models for investigating the detection and treatment of microbial keratitis, particularly when this might be due to two infective organisms.

Keywords Ex vivo cornea $\cdot$ Microbial keratitis · Colony-forming units $\cdot$ Corneal model

\section{Introduction}

Microbial keratitis is a major problem worldwide and is an important cause of vision loss and blindness. In vivo animal models, in vitro cell culture and ex vivo models have been used for investigating different aspects of this disease, including pathogenicity and treatment strategies [1-7].

In vivo studies require the use of a large number of animals to answer a research question. The welfare of these animals has become an important ethical issue [8], leading to the promotion of the philosophy of 'replacement, reduction and refinement' in the use of animals in research [9].

One way to overcome these issues is to use in vitro monolayer cultures of cells, including immortalised [5] or primary [4] corneal epithelial cells. However, these are not representative of the in vivo situation. They lack a three-dimensional (3D) structure and cross-talk between different epithelial cells, limbal cells and keratocytes. Consequently, advances have 
been made in 3D multi-layered tissue-engineered corneal constructs, and a few of these (EpiOcular ${ }^{\mathrm{TM}}$ from Mattek Corporation, Ashland, MA, USA, and HCE/corneal epithelium (SkinEthic) from Episkin, Lyon, France) are commercially available [10]. These have been used to study corneal pathogenesis $[6,11]$. Whilst they possess the $3 \mathrm{D}$ architecture of their in vivo counterparts, these models often use immortalised cell lines and lack intrinsic innate immune molecules which occur in vivo.

Recently, there has been some interest in the use of ex vivo corneal models to study keratitis $[12,13]$. Although these models lack immune elements, the $3 \mathrm{D}$ architecture remains, as do the intracellular innate immune molecules and cellularstromal components. These models have been used for studying wound healing [14], microbial adherence [12] and molecular microbial pathogenicity [13]. In our laboratory, we have used ex-vivo corneal models to study corneal epithelial regeneration $[15,16]$ and to develop models of inflammation. We have also shown that by gently rocking media over the corneas, they can be maintained in culture for at least 4 weeks [17].

To the best of our knowledge, a comparison of bacterial and fungal infections and of mixed infections has not been undertaken in ex vivo corneal models. We report a comparison of single- and mixed-species infections in both rabbit and human corneas to better understand the use of these models in microbial keratitis.

\section{Materials and methods}

\section{Materials}

We used corneas from two types of rabbits - wild brown rabbits (Blackface Meat Company, Dumfries, Scotland) and New Zealand rabbits (University of Sheffield, from rabbits sacrificed at the end of a licenced study). There was no difference in the performance of corneas from these two types of rabbits. Cadaveric human corneas unsuitable for transplant were acquired from the Ramayamma International Eye Bank, L V Prasad Eye Institute, Hyderabad, India. All corneas were obtained following procedures approved by the institutional review board for the protection of human subjects.

Dispase II was obtained from Roche Diagnostics (Burgess Hill, UK), and Videne ${ }^{\circledR}$ antiseptic solution was purchased from Ecolab (St. Paul, MN, USA). Mouse 3T3 fibroblasts (used in India) were from the American Type Culture Collection (ATCC; Manassas, VA, USA), and those used in the UK were an established J2 3T3 cell line originally from Professor Howard Green, USA. Epidermal growth factor was obtained from Invitrogen (Paisley, UK). For the culture of microorganisms, brain-heart infusion (BHI) agar and broth were purchased from Oxoid (Hampshire, UK) or HiMedia
(Mumbai, India). All other reagents were obtained from Sigma-Aldrich (Dorset, UK) unless otherwise stated. Calcofluor-white was obtained from Sigma-Aldrich (Dorset, UK) and from HiMedia (Mumbai, India).

\section{Isolation of rabbit corneas}

Corneas with sclera rims were dissected using a standard procedure including decontamination with povidone iodine, and were immediately placed into phosphate-buffered saline (PBS) [15].

\section{Ex vivo corneal organ culture}

Organ cultures were as previously described $[15,18]$. Rabbit and human corneoscleral buttons were placed epithelial side down in 35-mm petri dishes, and $500 \mu$ l Dulbecco's modified eagle's medium (DMEM)-agarose $(0.5 \% \mathrm{w} / \mathrm{v})$ solution was pipetted into the endothelial side of the cornea. The solution was allowed to solidify, and the buttons were then inverted so that the epithelium was facing up (Fig. 1a and b). Culture medium (DMEM: Ham's F12 [1:1] supplemented with $10 \%$ fetal calf serum [FCS], $100 \mathrm{U} \mathrm{ml}^{-1}$ penicillin and $100 \mathrm{U} \mathrm{ml}^{-1}$ streptomycin, $2.5 \mu \mathrm{g} \mathrm{ml}^{-1}$ amphotericin B, $5 \mu \mathrm{g} \mathrm{ml}^{-1}$ insulin and $10 \mathrm{ng} \mathrm{ml}^{-1}$ epidermal growth factor [EGF]) was added to submerge the ex vivo corneas. Prior to infection, corneas were washed three times with PBS and incubated in antibiotic- and antifungal-free medium for at least $24 \mathrm{~h}$ to remove residual antimicrobials.

All experimental work was performed on rabbit corneas in the UK and on human corneas in India.

\section{Culture of bacteria and fungi}

For rabbit corneas, laboratory strains of $S$. aureus (S-235), P. aeruginosa (SOM-1), C. albicans (SC5314) and F. solani strain (NCPF 2699), purchased from the National Collection of Pathogenic Fungi (UK), were used. For human corneas, ATCC cultures of $S$. aureus (25923), P. aeruginosa (27853) and $C$. albicans (90028) were used. All bacterial and fungal strains were cultured on brain-heart infusion (BHI) agar at $37{ }^{\circ} \mathrm{C}$ overnight and then maintained at $4{ }^{\circ} \mathrm{C}$. For use in experiments, one colony was sub-cultured from agar into BHI broth and incubated overnight at $37^{\circ} \mathrm{C}$. Stationary-phase microbes were used in rabbit cornea experiments. For human corneal experiments, on the day of corneal inoculation, a fresh broth was inoculated, and exponential-phase bacteria/fungi were used based on predetermined growth curves.

\section{Infection of ex vivo corneas}

Corneas were wounded with a scalpel (3 slashes vertically and 3 slashes horizontally), and a metal ring was placed on the 
Fig. 1 Schematic representation of a cross section (a) and a topdown image (b) of a corneoscleral button in organ culture. To infect corneas, a metal ring was placed on the corneoscleral button after wounding to form a seal, and bacteria/fungi were added to the surface of the cornea $(\mathbf{c})$

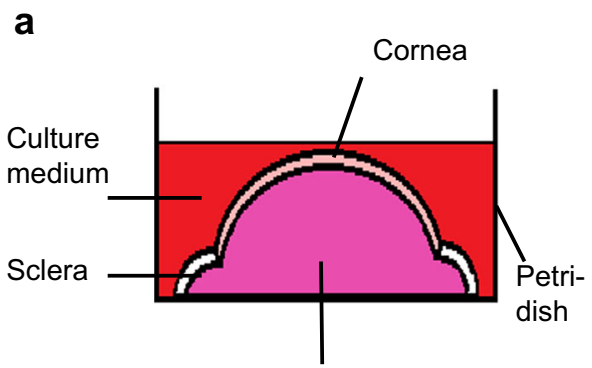

Agarose-culture medium

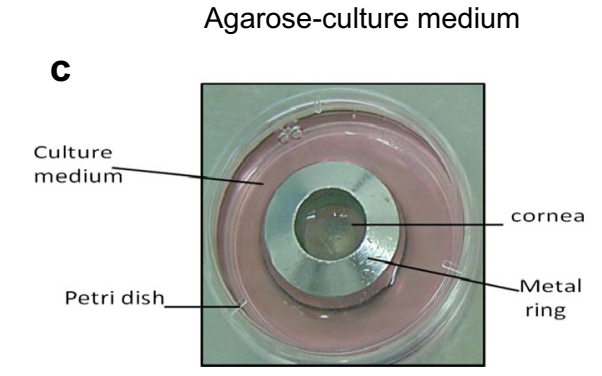

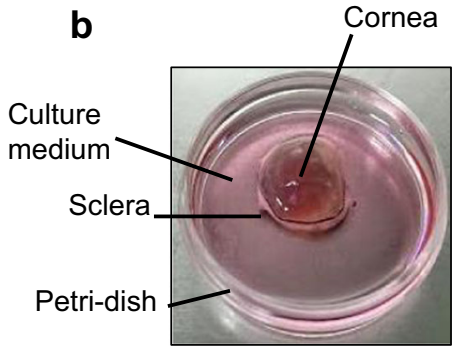

corneoscleral button, creating a watertight seal. Into the centre of the ring, $10^{8} \mathrm{~S}$. aureus, $P$. aeruginosa, C. albicans or F. solani were added (Fig. 1c), or the corneas were injected intrastromally (using a 26-gauge needle; Becton Dickinson, Oxford, UK) with the same number of organisms.

The infected corneas were incubated for 24 or $48 \mathrm{~h}$ at $37^{\circ} \mathrm{C}$, and were then homogenised and the resulting suspension serially diluted and spotted onto agar plates for colony enumeration. A set of infected corneas was also processed for histology and sections stained using Gram (bacteria) and periodic acid-Schiff (PAS) stains (fungi). Corneas not exposed to microbes were used as controls. Histological sections were imaged using a BX51 upright microscope and cell3D imaging software (Olympus, Essex, UK) in the UK or the ProgRes CapturePro 2.5 software (Jenoptik) in India.

\section{Imaging of microorganisms on the corneal surface}

To visualise bacteria, $10^{8} \mathrm{~S}$. aureus or P. aeruginosa were labelled using $1 \mathrm{mg} \mathrm{ml}^{-1}$ fluorescein isothiocyanate (FITC) for $1 \mathrm{~h}$ at $4{ }^{\circ} \mathrm{C}$, followed by four washes with PBS. For fungi, whole corneas were covered with 1:1 calcofluor white in 10\% $(v / v)$ potassium hydroxide for $10 \mathrm{~min}$ and washed three times with PBS. Bacteria- and fungi-infected corneas were imaged using fluorescence microscopy as described above.

\section{Statistical analysis}

Box-and-whisker plots of colony-forming units (CFU) per cornea were plotted using GraphPad Prism 6 software. All comparisons were analysed using Student's unpaired twotailed $t$ test, using Microsoft ${ }^{\circledR}$ Excel (Microsoft ${ }^{\circledR}$ Office, 2010). A $p$ value $\leq 0.05$ was considered significant.

\section{Results}

\section{Macroscopic view of rabbit and human corneas}

Corneas infected with bacteria and fungi showed a visible increase in haze compared with uninfected corneas (Fig. 2). The scratches were visible in all corneas, but were more evident in infected corneas than uninfected corneas (Fig. 2).

FITC-labelled bacteria within infected rabbit and human corneas are shown in Fig. 3. It was observed that $S$. aureus cells covered the surface of the cornea at $24 \mathrm{~h}$ and $48 \mathrm{~h}$, and at certain locations, clumps of bacteria ranging from 5 to $25 \mu \mathrm{m}$ in diameter were detected. On the other hand P. aeruginosainfected corneas showed fewer clumps than observed with S. aureus, after 24 and $48 \mathrm{~h}$. A microscopic view of the surface of calcofluor white-stained C. albicans-infected corneas showed a more uniform spread of yeast and a few hyphal forms on the surface of the cornea after $24 \mathrm{~h}$, which increased in both the distribution of individual yeast cells and the spread of hyphae by $48 \mathrm{~h}$ (Fig. 3). The distribution of F. solani at $24 \mathrm{~h}$ was more punctuated with hyphae at distinct places in both rabbit and human corneas (Fig. 3). After 48 h, the surface of the cornea was covered with a mat of fungi, where the hyphae could be observed extending into the scratch and in all directions, away from the fungal bulk (Fig. 3). The coverage of bacteria and fungi over the corneal surface was similar between rabbit and human corneas.

\section{Single-species infection of rabbit and human corneas}

After $24 \mathrm{~h}, \mathrm{CFU}$ recovered per cornea for $S$. aureus, $P$. aeruginosa, $C$. albicans and $F$. solani were as follows (Fig. 4): $5.1 \pm 1.0 \times 10^{5}, 1.9 \pm 0.3 \times 10^{7}, 3.0 \pm 0.6 \times 10^{5}$ and $2.5 \pm 0.9 \times 10^{5} \mathrm{CFU} /$ rabbit cornea, respectively, and $3.8 \pm$ 
Fig. 2 Fluorescein-stained rabbit and human corneas showing turbidity of infected versus noninfected corneas. Corneas were scalpel-wounded and exposed to $S$. aureus, $P$. aeruginosa, C. albicans or F. solani for $24 \mathrm{~h}$. Corneas were briefly washed and stained with $0.5 \mathrm{mg} \mathrm{ml}^{-1}$ of fluorescein isothiocyanate for $30 \mathrm{~min}$, washed again and photographed. Arrows indicate scalpel wounds

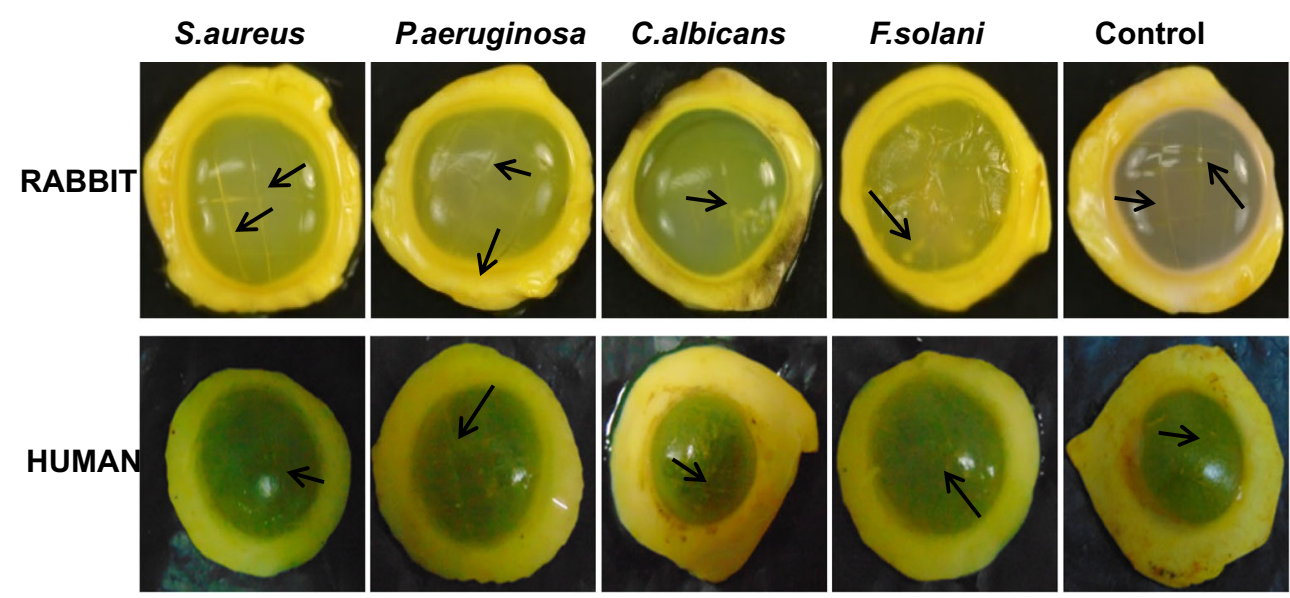

$0.8 \times 10^{6}, 4.4 \pm 0.6 \times 10^{8}, 1.9 \pm 0.3 \times 10^{5}$ and $1.8 \pm 0.1 \times 10^{3}$ $\mathrm{CFU} /$ human cornea, respectively. A significantly higher number of $S$. aureus and $P$. aeruginosa were recovered after $48 \mathrm{~h}$ of incubation in both rabbit $\left(1.7 \pm 0.3 \times 10^{6}(p=0.00005), 4.4\right.$ $\pm 0.7 \times 10^{7}(p=0.0009)$ and human corneas $\left(1.5 \pm 0.4 \times 10^{7}\right.$ $(p=0.0004), 6.5 \pm 3.0 \times 10^{8}(p=0.0057)$, respectively, compared to yields at $24 \mathrm{~h}$. There was no significant difference in the recovery of $C$. albicans or $F$. solani after 48 h, with $5.1 \pm$ $1.5 \times 10^{5}(p=0.159)$ and $1.6 \pm 0.7 \times 10^{6}(p=0.090) \mathrm{CFU} /$ rabbit cornea, respectively, and $5.3 \pm 1.6 \times 10^{5}(p=0.108)$ and $2.1 \pm 0.1 \times 10^{3}(p=0.081) \mathrm{CFU} /$ human cornea, respectively. In addition, there was approximately tenfold greater recovery of bacteria from human corneas than from rabbit corneas after both 24 and $48 \mathrm{~h}$.

The injection method involved the introduction of bacteria and fungi into the stroma. Compared to the scalpel method, after $24 \mathrm{~h}$, injection of a single-species organism resulted in higher $\mathrm{CFU} /$ cornea $(p<0.05)$ for all organisms, with the exception of $C$. albicans in human corneas, where no significant difference was observed $(p=0.057)$.
Fig. 3 FITC-labelled S. aureus or $P$. aeruginosa were incubated with scalpel wounded rabbit (a) and human (b) corneas for 24 and $48 \mathrm{~h}$, washed and imaged using a fluorescent microscope. Unlabelled C. albicans and F. solani were incubated with rabbit and human corneas for 24 and $48 \mathrm{~h}$, washed, the model stained with Calcofluor White and imaged using a fluorescent microscope. The distribution of bacteria and fungi over the surface of the cornea and located within the scratch wound can be observed a

$24 \mathrm{~h}$

$48 \mathrm{~h}$

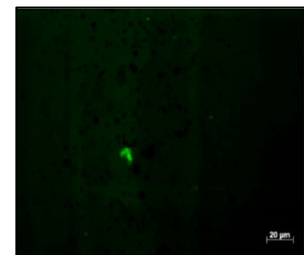

b

$24 h$
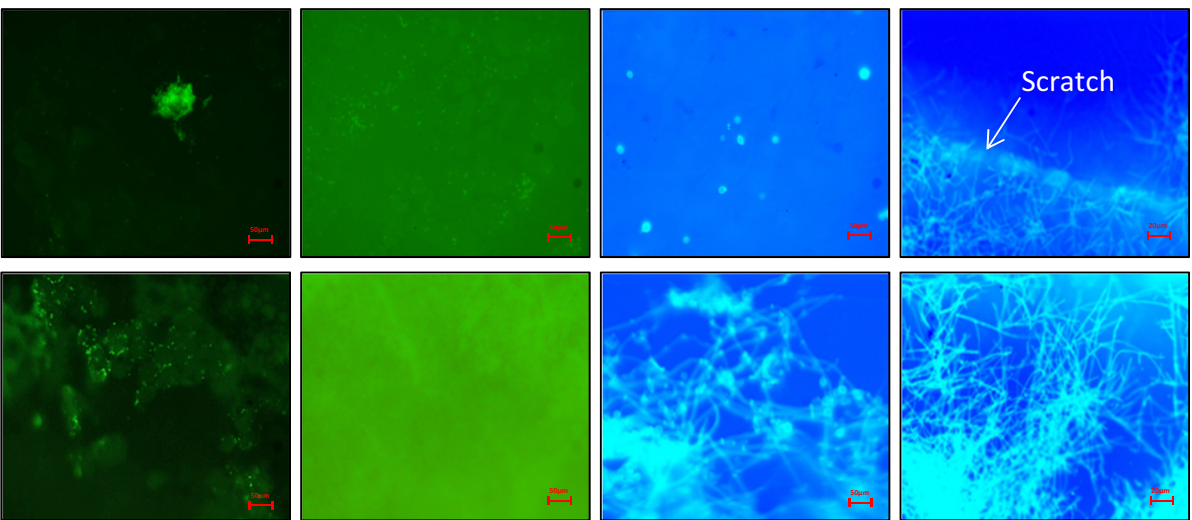

P.aeruginosa
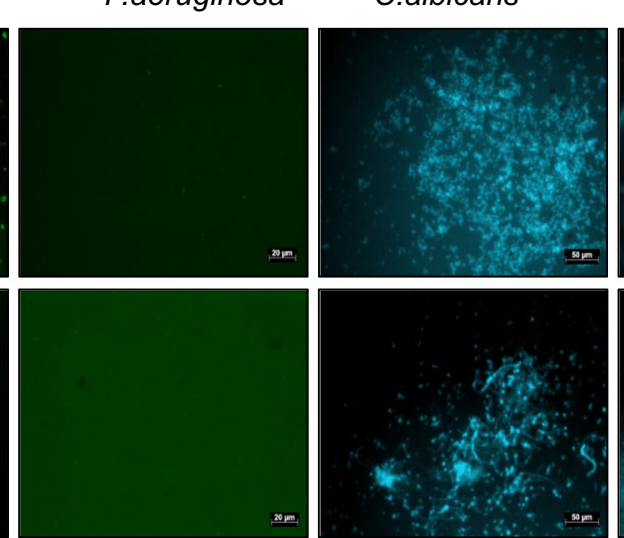

\section{F.solani}
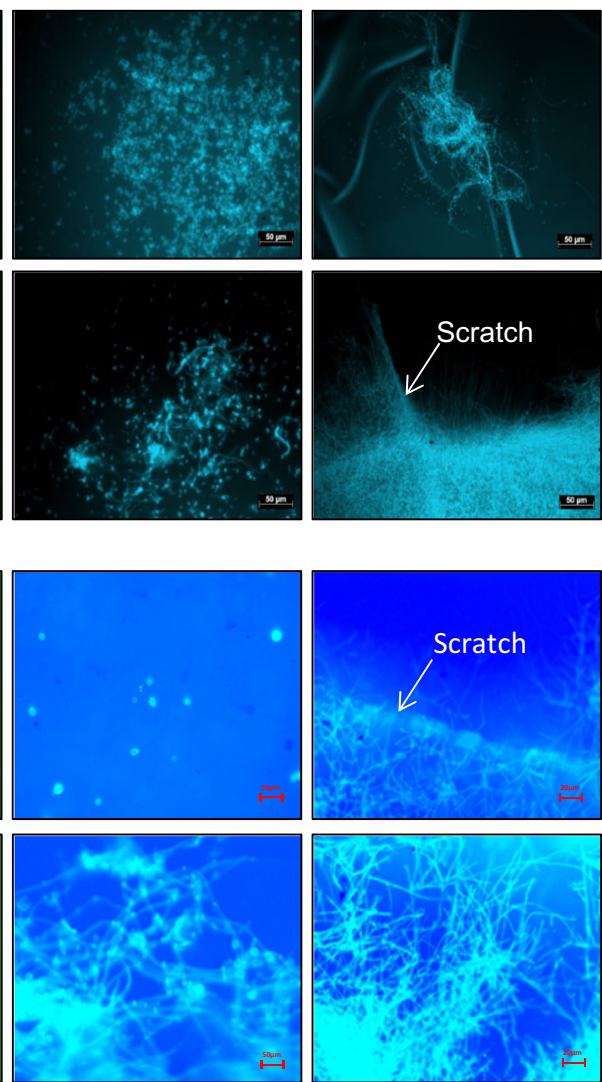


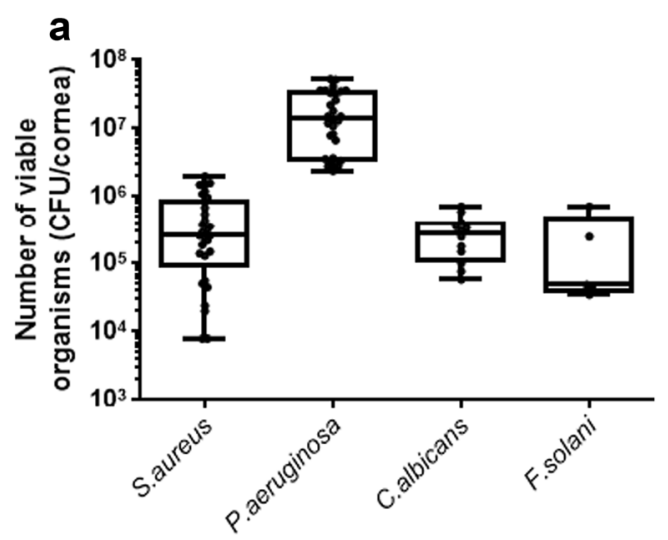

(Fig. 5a), which increased after $48 \mathrm{~h}$ in both number and depth of penetration.

\section{Two-species infection of corneas}

In the two-species infection model, we were able to recover both bacterial species from infected rabbit and human corneas (Table 1). Histological examination confirmed the quantitative colony count data (Fig. 6). As with the single-species model, infiltration of $P$. aeruginosa cells was found throughout the stroma, including Descemet's membrane, whereas $S$. aureus showed little spread beyond the injection site.

A mixed infection involving C. albicans and P. aeruginosa

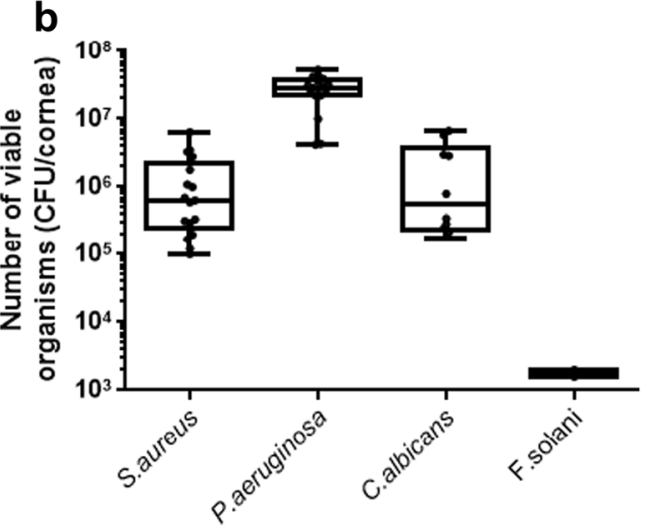
is the most commonly observed clinically [19]. In ex vivo models of this mixed infection, both organisms were recovered after $24 \mathrm{~h}$ following scalpel wounding, with $P$. aeruginosa showing dominance within the tissue by both colony counting $\left(4.20 \pm 1.6 \times 10^{6}\right.$ and $2.12 \pm 0.9 \times 10^{8} \mathrm{CFU} /$ rabbit cornea and $5.15 \pm 6.6 \times 10^{5}$ and $1.00 \pm 1.2 \times 10^{8} \mathrm{CFU} /$ human cornea for C. albicans and P. aeruginosa, respectively) and histology (Table 1 and Fig. 6). The level of recovery of both organisms was approximately the same regardless of the method of introduction of organisms.

Fig. 4 Single-species infection of ex vivo rabbit (a) and human (b) corneas. Ex vivo corneas were scratched six times with a scalpel and exposed to single-species inoculum of $S$. aureus, P. aeruginosa, C. albicans or $F$. solani for $24 \mathrm{~h}$ at $37{ }^{\circ} \mathrm{C}$. The models were then homogenised, and the resulting suspension serially diluted and plated onto agar plates. The number of colony-forming units per cornea were plotted for each cornea. The box plot shows the minimum and maximum values depicted by the bars; the upper quartile, median and lower quartile are depicted by the top, middle and bottom horizontal lines, respectively

The histology of single-species-infected corneas after $24 \mathrm{~h}$ is shown in Fig. 5a. Here, vast infiltration of P. aeruginosa can be seen covering the epithelium and entire stroma and infiltrating the Descemet's membrane. This was independent of the method of inoculation (data not shown). The histology of the $S$. aureus-infected corneas was characterised by the concentration of the majority of organisms within the scratches (Fig. 5a). The number of bacteria within tissue sections correlated with the CFU/cornea data (Fig. 4).

The distribution of $C$. albicans within the corneal tissue was similar between the human and rabbit corneas. Yeast cells and hyphal elements were observed close to the scratch site, with no infiltration beyond $150 \mu \mathrm{m}$ into the stroma (Fig. 5a). The number of hyphae and the infiltration of $C$. albicans cells into the stroma after $48 \mathrm{~h}$ did not differ from that observed after $24 \mathrm{~h}$.

The tissue penetration by $F$. solani after scalpel wounding was less than that for $C$. albicans at $24 \mathrm{~h}$, with infiltration not more than $10 \mu \mathrm{m}$ into the stroma from the site of inoculation

\section{Discussion}

Ex vivo models have previously been used to study cornealmicrobial interactions $[12,13]$. However, there have been no direct comparisons of single and mixed bacterial and fungal infections or a comparison of the infection of rabbit and human corneas. Here, we describe the numbers of viable organisms recovered from rabbit and human corneas after 24 and $48 \mathrm{~h}$, showing histological images from scalpel wounding and intrastromal injection as ways of introducing organisms to the cornea.

A variety of methods have been described in the literature for introducing bacteria or fungi to experimental (in vivo, in vitro or ex vivo) corneas. These include the use of bacterial/fungal-inoculated contact lenses [20,21], blotting paper and ethylene glycol tetraacetic acid (EGTA) [7], and mechanical removal of the epithelial surface $[22,23]$. However, the most commonly described methods are corneal scratch $[24,25]$ and intrastromal injection [26, 27]. Therefore, we chose to scratch the corneas with a scalpel six times, so that the scratch revealed the upper stromal compartment, and also to introduce organisms intrastromally using an injection method. Although the injection method gave a greater yield of organisms than the scalpel method, we observed that the scalpel method mimicked clinical infection in which infection is initiated from an abrasion on the corneal surface [28]. Because it was thought that the infiltrative capacity of $P$. aeruginosa within the tissue might inhibit or prevent the growth and 
a
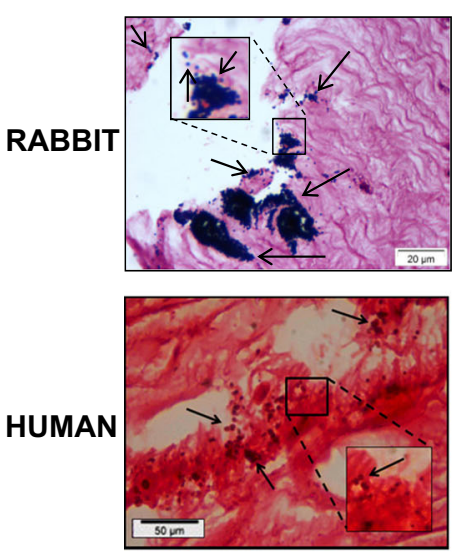

b
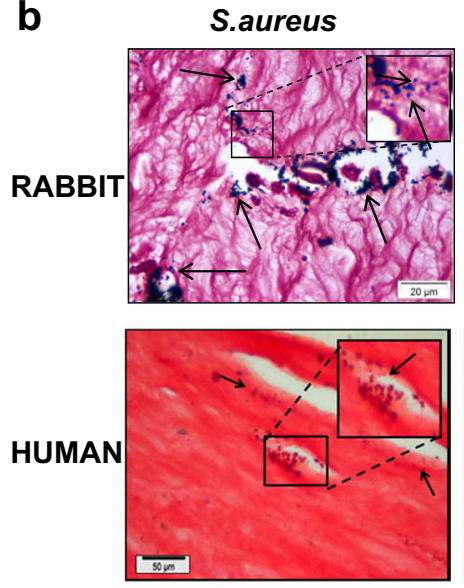

P.aeruginosa
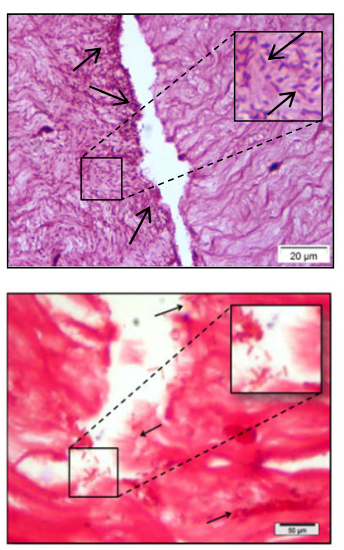

P.aeruginosa
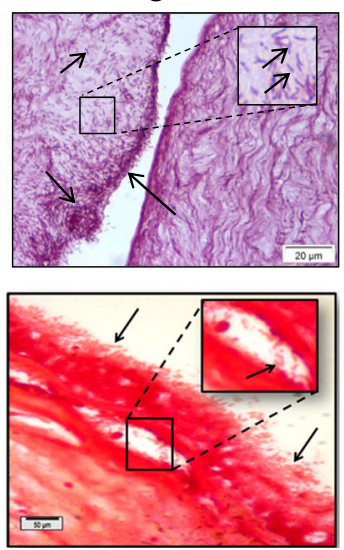

C.albicans
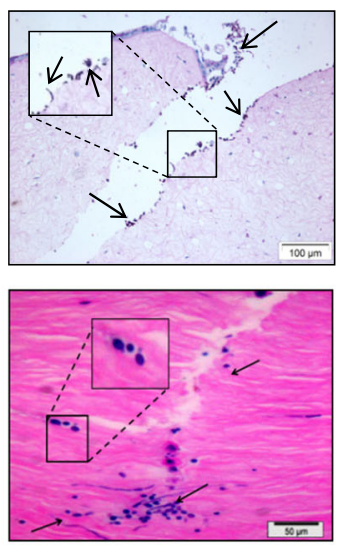

C.albicans
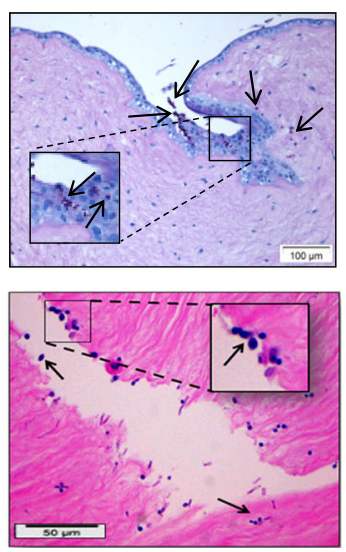

F.solani
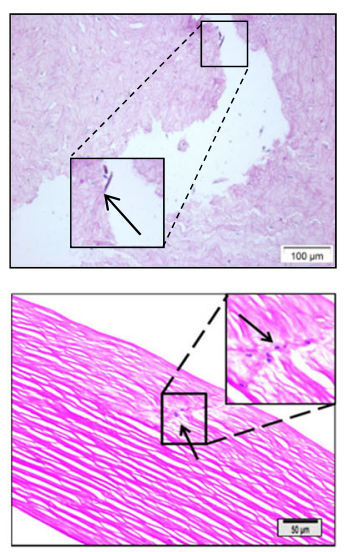

F.solani
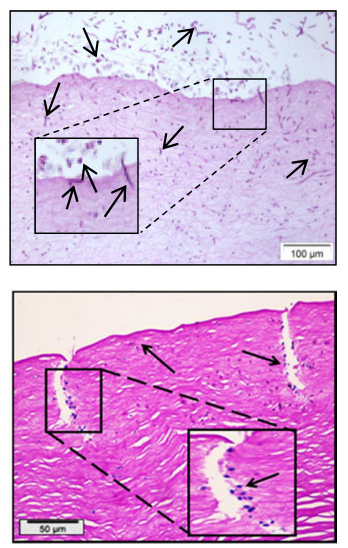

Fig. 5 Histology of single-species infection of ex vivo rabbit and human corneas. Ex vivo rabbit and human corneas were scratched six times with a scalpel and exposed to a single-species inoculum of $S$. aureus, P. aeruginosa, C. albicans or F. solani for $24 \mathrm{~h}$ (a) or $48 \mathrm{~h}$ (b). Corneas were fixed in $10 \%$ buffered formalin, embedded in paraffin, sectioned and stained using Gram stain (S. aureus and P. aeruginosa) or PAS stain (C. albicans and F. solani). Gram-positive (purple) cocci (S. aureus), Gram-negative (pink) rods ( $P$. aeruginosa), and purple round yeast and hyphae (C. albicans and $F$. solani) can be observed at the epithelial surface and within the scratch, and are present in the stroma

Table 1 Numbers of CFU/cornea recovered from multi-bacterial/fungal infections after $24 \mathrm{~h}$

\begin{tabular}{|c|c|c|c|c|}
\hline & RABBIT & & HUMAN & \\
\hline \multirow[t]{2}{*}{ S. aureus - P. aeruginosa } & S. aureus & P. aeruginosa & S. aureus & P. aeruginosa \\
\hline & $1.88 \pm 0.6 \times 10^{6}$ & $3.09 \pm 0.9 \times 10^{7}$ & $5.25 \pm 1.6 \times 10^{5}$ & $2.43 \pm 3.2 \times 10^{8}$ \\
\hline \multirow[t]{2}{*}{ C. albicans - P. aeruginosa } & C. albicans & P. aeruginosa & C. albicans & P. aeruginosa \\
\hline & $4.20 \pm 1.6 \times 10^{6}$ & $2.12 \pm 0.9 \times 10^{8}$ & $5.15 \pm 6.6 \times 10^{5}$ & $1.00 \pm 1.2 \times 10^{8}$ \\
\hline \multicolumn{5}{|l|}{ B } \\
\hline & RABBIT & & HUMAN & \\
\hline \multirow[t]{2}{*}{ S. aureus - $P$. aeruginosa } & S. aureus & P. aeruginosa & S. aureus & P. aeruginosa \\
\hline & $9.26 \pm 4.2 \times 10^{6}$ & $5.83 \pm 2.4 \times 10^{8}$ & $2.50 \pm 5.7 \times 10^{4}$ & $3.72 \pm 1.08 \times 10^{8}$ \\
\hline \multirow[t]{2}{*}{ C. albicans - P. aeruginosa } & C. albicans & P. aeruginosa & C. albicans & P. aeruginosa \\
\hline & $4.25 \pm 1.1 \times 10^{6}$ & $6.84 \pm 0.6 \times 10^{8}$ & $4.05 \pm 9.6 \times 10^{5}$ & $7.65 \pm 1.0 \times 10^{8}$ \\
\hline
\end{tabular}

Two multi-pathogen infections were investigated. These were a mixed S. aureus/P. aeruginosa and a mixed C. albicans/P. aeruginosa infection. A: EX vivo rabbit and human corneas were wounded with a scalpel and exposed to a mixture of $10^{8}$ of both organisms for $24 \mathrm{~h}$ B: Ex vivo rabbit and human corneas were intrastromally injected with $10^{8}$ cells of the first organism at 3-5 distinct locations, and then $10^{8}$ cells of the second organism were similarly injected at different sites. The corneas were incubated for $24 \mathrm{~h}$, washed, homogenised, serially diluted and plated onto agar plates, and the CFU/ cornea calculated. Data is expressed in CFU/cornea \pm SEM of at least three independent experiments performed in triplicate 


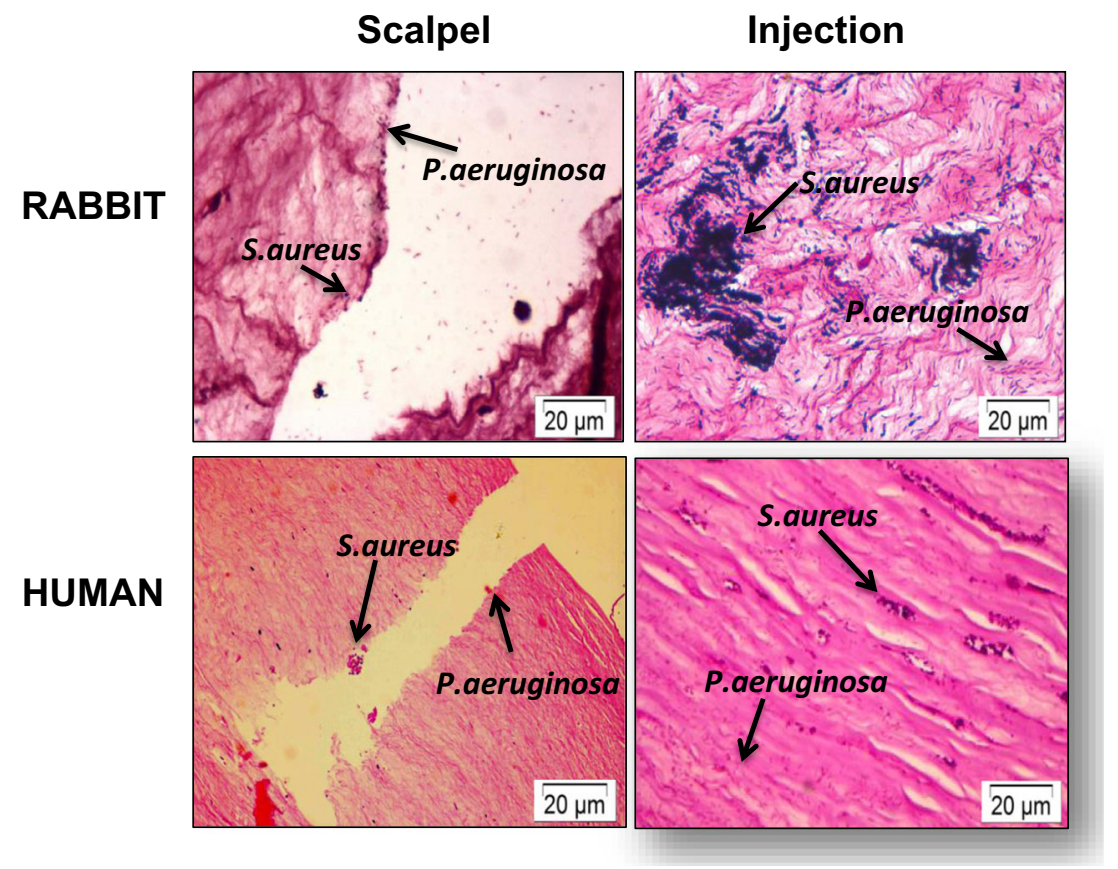

Fig. 6 Histology of rabbit and human ex vivo models showing a mixed $S$. aureus and $P$. aeruginosa infection. At different sites within the same cornea, ex vivo corneas were intrastromally injected with $10^{8} \mathrm{~S}$. aureus and $10^{8}$ P. aeruginosa and incubated for $24 \mathrm{~h}$ (injection). Alternatively, corneas were wounded with a scalpel, and $10^{8} \mathrm{~S}$. aureus and $10^{8}$

propagation of $S$. aureus and/or C. albicans when introduced together through a scalpel wound, we compared this method with intrastromal injection, injecting organisms at separate sites and thus preventing their interaction. However, the scalpel method did not prevent the recovery of $S$. aureus or C. albicans, suggesting that either method is suitable for establishing a mixed infection model. Therefore, we show that an infection can be induced in the ex vivo corneas for both single and multiple species using either scalpel wounding or intrastromal injection.

The following aspects of this model need further discussion:

1. A large bacterial/fungal inoculum was used, because this corneal model does not have a blood supply or immune system. Consequently, the damage that the inflammation causes to the local tissue, and which provides additional nutrients via the vasculature for the bacteria/fungi, was not present.

2. S. aureus was not typically found at the epithelial surface, but rather within the scratches, commonly in clusters, and not migrating into surrounding and deeper cornea. This has been described previously as well $[29,30]$. The observed attachment of $S$. aureus at the stromal surface is also supported by the observations of Rhem et al. [29], who demonstrated that collagen-binding clinical $S$. aureus isolates expressing the cna collagen-binding gene showed
P. aeruginosa were added to the surface of the cornea for $24 \mathrm{~h}$ (scalpel). Sections were Gram-stained and imaged to visualise $S$. aureus and $P$. aeruginosa within their injection sites at distinct locations within the stroma. P. aeruginosa shows widespread infiltration into the tissue, whereas $S$. aureus shows less infiltration

enhanced tissue disruption compared to a $\mathrm{cna}^{-}$isogenic mutant. The cna protein is considered to be a virulence factor mediating bacterial adherence to the epithelial surface and the stroma, and neutrophil recruitment to the infection site. Of the two strains of $S$. aureus that we used in this study, the ATCC 25923 strain is known to express this gene [31], which could be the reason for the higher level of binding within the scratch than at the surface (it is unknown whether this gene is expressed in the local clinical strain, S235). According to reports in the literature, in vivo corneas infected with $S$. aureus by intrastromal injection returned bacterial counts of approximately $10^{4}-10^{7} \mathrm{CFU} /$ cornea $[32,33]$, depending on the number of bacteria in the starting inoculum and the length of time the bacteria were incubated with the eye. These values are in line with the recovery we obtained from ex vivo models, suggesting that our model is representative of an in vivo infection in terms of the number of bacteria recovered.

3. We did not observe any ulceration or corneal edema. This is because ex vivo corneas lack inflammatory cells that are primarily responsible for epithelial ulceration [32], stromal polymorphonuclear neutrophil (PMN) infiltration [34-36], and ulcer formation [37] seen in clinical S. aureus infection.

4. Compared to the $S$. aureus model, P. aeruginosa-infected corneas yielded a greater number of $\mathrm{CFU} /$ cornea and were 
seen infiltrating the entire cornea, despite having the same inoculum. This high level of infiltration has been shown to be the result of proteolytic bacterial enzymes, including type III secretion system-associated cytotoxins, exoenzyme U and exoenzyme S [38, 39], alkaline protease and elastase [40], which have been shown to contribute to corneal erosion [41]. In addition, host proteolytic enzymes also contribute to corneal ulceration [26]. We observed a softening of $P$. aeruginosa-infected corneas and increased opacity compared with control corneas, but no ulceration. As mentioned previously, this was due to the lack of an immune cell component in these ex vivo cultures.

5. Previous studies have reported an increase in the recovery of $P$. aeruginosa from corneas compared with the initial inoculum [42, 43]. However, we did not find this increased recovery of $P$. aeruginosa. Although we have no definitive explanation for this observation, one possible explanation is that only a portion of these organisms actually adhere to the corneal surface and are able to invade/ colonise. The data presented suggest that $10^{6}-10^{7} \mathrm{CFU} /$ cornea is the maximum number that can be recovered from an ex vivo cornea, and this maximal amount occurs after $24 \mathrm{~h}$.

6. In contrast to the bacterial infections, single-species infections with $C$. albicans and $F$. solani did not show a significant increase in the recovery of organisms after $48 \mathrm{~h}$ versus $24 \mathrm{~h}$, and the numbers of organisms recovered were lower, despite the same inoculum. This has been described briefly in the literature, where as many as $10^{9}-10^{6} \mathrm{CFU} /$ cornea for C. albicans and $10^{3} \mathrm{CFU} /$ cornea for $F$. solani were inoculated into in vivo or ex vivo murine, rabbit or rat models, with recovery of as little as $10^{5}-10^{3}$ C. albicans CFU/cornea [12, 44-46], and $10^{3}$ F. solani CFU/cornea [47], respectively. The reason for this is not fully understood.

7. From the 24-h histology images presented here, little infiltration of fungi into the corneal tissue is seen, with organisms remaining predominantly at the surface. However, particularly for $F$. solani, there was vast infiltration of fungal cells throughout the stroma after $48 \mathrm{~h}$. The histology of infected in vivo cultures mimics histological images of clinical infection, with a dense white fungal plaque, corneal opacity, corneal infiltration, oedema, ulcer formation, satellite lesions, corneal neovascularisation and hypopyon [47-51]. Yeast forms of C. albicans and conidia of $F$. solani $i$ are shown to adhere to the stroma, and after a period of time, hyphae form that penetrate the stroma [12, 45, 52] to a depth of approximately $150 \mu \mathrm{m}$ [44]. This was observed in our ex vivo rabbit and human cornea models.

8. Differences were observed between rabbit and human corneas primarily in the number of organisms recovered from each cornea, i.e. there was an approximately tenfold increase in the recovery of bacteria from human versus rabbit corneas. This may have been due to the use of different bacterial strains (ATTC strains [human] and local clinical strains [rabbit]), intrinsic differences between the corneas of the two species, including anatomical and molecular differences such as differences in the Bowman's membrane [44] arrangement of collagen fibres [53], size, thickness [54], secretion of antimicrobial peptides [55], or surface mucin modifications [56]. Furthermore, the difference in bacterial recovery could be due to the use of stationary-phase organisms in rabbit experiments and log-phase bacteria in human corneal experiments. However, in comparing these two types of inocula, we have established that a similar number of bacteria in either phase still results in a clinically relevant level of infection for both single- and multi-species infection in both corneas, showing comparable histology.

9. The length of time these models were cultured in vitro was short. The acute nature of such an infection limits its use to short-term experiments involving, for example, investigation of treatment strategies [57], innate immune responses [58], detection of organisms [59] and host-microbe interactions [18]. These models were not intended to replicate the clinical outcome of infection that can be observed in vivo, which may develop over several weeks when not treated effectively. In these ex vivo models, there is an obvious lack of a host immune component, and the presence and infiltration of inflammatory cells is thought to contribute to the severity of disease [26]. As such, these ex vivo models do not form corneal ulcers as typically observed clinically [18, 41, 48]. They also lack tear films, which play a defensive role.

In summary, we achieved our aim of establishing a reproducible infection of both human and animal corneas. It is certain that no model system (including animals) is a perfect surrogate for the natural human infection. Nonetheless, useful data can still be obtained. We show that we can establish a reproducible in vitro bacterial and fungal infection, with the final number of recoverable bacteria/fungi comparable to that from natural in vivo experiments. These models are now being used in the evaluation of microbial detection systems.

\section{Compliance with ethical standards}

Human and animal rights and informed consent This article does not contain any studies with human participants or animals performed by any of the authors.

Funding The Wellcome Trust provided financial support in the form of a grant through the Affordable Healthcare in India Initiative (no. 0998800/B/12/Z). The sponsor had no role in the design or conduct of this research. 
Conflict of interest All authors certify that they have no affiliations with or involvement in any organization or entity with any financial interest (such as honoraria; educational grants; participation in speakers' bureaus; membership, employment, consultancies, stock ownership, or other equity interest; and expert testimony or patent-licensing arrangements) or non-financial interest (such as personal or professional relationships, affiliations, knowledge or beliefs) in the subject matter or materials discussed in this manuscript.

Open Access This article is distributed under the terms of the Creative Commons Attribution 4.0 International License (http:// creativecommons.org/licenses/by/4.0/), which permits unrestricted use, distribution, and reproduction in any medium, provided you give appropriate credit to the original author(s) and the source, provide a link to the Creative Commons license, and indicate if changes were made.

\section{References}

1. Chandra J, Pearlman E, Ghannoum M (2014) Animal models to investigate fungal biofilm formation. Methods Mol Biol 1147:141157. doi:10.1007/978-1-4939-0467-9 10

2. Evans DJ, Fleiszig SM (2013) Why does the healthy cornea resist Pseudomonas aeruginosa infection? Am J Ophthalmol 155:961970. doi:10.1016/j.ajo.2013.03.001

3. Yang K, Wu M, Li M, Li D, Peng A, Nie X, Sun M, Wang J, Wu Y, Deng Q (2014) miR-155 suppresses bacterial clearance in Pseudomonas aeruginosa keratitis by targeting Rheb. J Infect Dis 89-98. doi: 10.1093/infdis/jiu002

4. Zaidi T, Zaidi T, Yoong P, Pier GB (2013) Staphylococcus aureus corneal infections: effect of the Panton-valentine leukocidin (PVL) and antibody to PVL on virulence and pathology. Invest Ophthalmol Vis Sci 54:4430-4438. doi:10.1167/iovs.13-11701

5. Kolar SSN, Luca V, Baidouri H, Mannino G, McDermott AM, Mangoni ML (2014) Esculentin-1a (1-21) NH2: a frog skinderived peptide for microbial keratitis. Cell Mol Life Sci 1-11. doi: 10.1007/s00018-014-1694-0

6. Alarcon I, Evans DJ, Fleiszig SM (2009) The role of twitching motility in Pseudomonas aeruginosa exit from and translocation of corneal epithelial cells. Invest Ophthalmol Vis Sci 50:22372244. doi: $10.1167 /$ iovs.08-2785

7. Alarcon I, Tam C, Mun JJ, LeDue J, Evans DJ, Fleiszig SM (2011) Factors impacting corneal epithelial barrier function against Pseudomonas aeruginosa traversal. Invest Ophthalmol Vis Sci 52:1368-1377. doi:10.1167/iovs.10-6125

8. Badyal DK, Desai C (2014) Animal use in pharmacology education and research: the changing scenario. Indian J Pharm 46:257-265. doi:10.4103/0253-7613.132153

9. Editorial (2010) Reduce, refine, replace. Nat Immunol 11:971. doi: 10.1038/ni1110-971

10. Shah A, Brugnano J, Sun S, Vase A, Orwin E (2008) The development of a tissue-engineered cornea: biomaterials and culture methods. Pediatr Res 63:535-544. doi:10.1203/PDR.0b013 e31816bdf54

11. Augustin DK, Heimer SR, Tam C, Li WY, Le Due JM, Evans DJ, Fleiszig SM (2011) Role of defensins in corneal epithelial barrier function against Pseudomonas aeruginosa traversal. Infect Immun 79:595-605. doi:10.1128/iai.00854-10

12. Zhou Q, Chen H, Qu M, Wang Q, Yang L, Xie L (2011) Development of a novel ex vivo model of corneal fungal adherence. Graefes Arch Clin Exp Ophthalmol 249:693-700. doi:10.1007/s00417-010-1601-9
13. Hua X, Yuan X, Di Pietro A, Wilhelmus KR (2010) The molecular pathogenicity of Fusarium keratitis: a fungal transcriptional regulator promotes hyphal penetration of the cornea. Cornea 29:1440 1444. doi:10.1097/ICO.0b013e3181d8383a

14. Castro-Combs J, Noguera G, Cano M, Yew M, Gehlbach PL, Palmer J, Behrens A (2008) Corneal wound healing is modulated by topical application of amniotic fluid in an ex vivo organ culture model. Exp Eye Res 87:56-63. doi:10.1016/j.exer.2008.04.010

15. Deshpande P, Notara M, Bullett N, Daniels JT, Haddow DB, MacNeil S (2009) Development of a surface-modified contact lens for the transfer of cultured limbal epithelial cells to the cornea for ocular surface diseases. Tissue Eng A 15:2889-2902. doi:10.1089 /ten.TEA.2008.0528

16. Ortega Í, Deshpande P, Gill AA, MacNeil S, Claeyssens F (2013) Development of a microfabricated artificial limbus with micropockets for cell delivery to the cornea. Biofabrication 5: 025008. doi: $10.1088 / 1758-5082 / 5 / 2 / 025008$

17. Deshpande P, Ortega Í, Sefat F, Sangwan VS, Green N, Claeyssens F, MacNeil S (2015) Rocking media over ex vivo corneas improves this model and allows the study of the effect of proinflammatory cytokines on wound healing. Invest Ophthalmol Vis Sci 56:15531561. doi:10.1167/iovs.14-15308

18. Alekseev O, Tran AH, Azizkhan-Clifford J (2012) Ex vivo organotypic corneal model of acute epithelial herpes simplex virus type I infection. J Vis Exp e3631. doi: 10.3791/3631

19. Ray M, Nigel LC, Tan AM (2014) Triple infection keratitis. Eye Contact Lens 40:123-126. doi:10.1097/icl.0000000000000022

20. Lawin-Brussel CA, Refojo MF, Leong FL, Hanninen L, Kenyon KR (1993) Effect of Pseudomonas aeruginosa concentration in experimental contact lens-related microbial keratitis. Cornea 12: $10-18$

21. Cole N, Hume EB, Vijay AK, Sankaridurg P, Kumar N, Willcox MD (2010) In vivo performance of melimine as an antimicrobial coating for contact lenses in models of CLARE and CLPU. Invest Ophthalmol Vis Sci 51:390-395. doi:10.1167/iovs.09-4068

22. Stangogiannis-Druya E, Stangogiannis-Druya C, Naranjo-Tackman R, Vanzzini V, Villar-Kuri J (2009) Bacterial corneal ulcer treated with intrastromal antibiotic. Experimental model in vivo. Arch Soc Esp Oftalmol 84:123-132

23. Sun Y, Hise AG, Kalsow CM, Pearlman E (2006) Staphylococcus aureus-induced corneal inflammation is dependent on Toll-like receptor 2 and myeloid differentiation factor 88. Infect Immun 74: 5325-5332. doi:10.1128/iai.00645-06

24. Blaylock WK, Yue BY, Robin JB (1990) The use of concanavalin A to competitively inhibit Pseudomonas aeruginosa adherence to rabbit corneal epithelium. CLAO J 16:223-227

25. Kwong MS, Evans DJ, Ni M, Cowell BA, Fleiszig SM (2007) Human tear fluid protects against Pseudomonas aeruginosa keratitis in a murine experimental model. Infect Immun 75:2325-2332. doi:10.1128/iai.01404-06

26. Kessler E, Mondino BJ, Brown SI (1977) The corneal response to Pseudomonas aeruginosa: histopathological and enzymatic characterization. Invest Ophthalmol Vis Sci 16:116-125

27. Barequet IS, Bourla N, Pessach YN, Safrin M, Yankovich D, Ohman DE, Rosner M, Kessler E (2012) Staphylolysin is an effective therapeutic agent for Staphylococcus aureus experimental keratitis. Graefes Arch Clin Exp Ophthalmol 250:223-229. doi:10.1007/s00417-011-1822-6

28. Deorukhkar S, Katiyar R, Saini S (2012) Epidemiological features and laboratory results of bacterial and fungal keratitis: a five-year study at a rural tertiary-care hospital in western Maharashtra, India. Singapore Med J 53:264-267

29. Rhem MN, Lech EM, Patti JM, McDevitt D, Hook M, Jones DB, Wilhelmus KR (2000) The collagen-binding adhesin is a virulence factor in Staphylococcus aureus keratitis. Infect Immun 68:37763779 
30. Hume EB, Dajcs JJ, Moreau JM, Sloop GD, Willcox MD, O'Callaghan RJ (2001) Staphylococcus corneal virulence in a new topical model of infection. Invest Ophthalmol Vis Sci 42: 2904-2908

31. Almeida LM, de Almeida MZP, Mendonça CL, Mamizuka EM (2013) Comparative analysis of agr groups and virulence genes among subclinical and clinical mastitis Staphylococcus aureus isolates from sheep flocks of the Northeast of Brazil. Braz J Microbiol 44:493-498. doi:10.1590/S1517-83822013000200026

32. Oguz H, Ozbilge H, Oguz E, Gurkan T (2005) Effectiveness of topical taurolidine versus ciprofloxacin, ofloxacin, and fortified cefazolin in a rabbit Staphylococcus aureus keratitis model. Curr Eye Res 30:155-161. doi:10.1080/02713680490908733

33. Barequet IS, Ben Simon GJ, Safrin M, Ohman DE, Kessler E (2004) Pseudomonas aeruginosa LasA protease in treatment of experimental staphylococcal keratitis. Antimicrob Agents Chemother 48:1681-1687

34. Hume EB, Cole N, Khan S, Garthwaite LL, Aliwarga Y, Schubert TL, Willcox MD (2005) A Staphylococcus aureus mouse keratitis topical infection model: cytokine balance in different strains of mice. Immunol Cell Biol 83:294-300. doi:10.1111/j.14401711.2005.01326.x

35. Hsiao CH, Ong SJ, Chuang CC, Ma DH, Huang YC (2015) A comparison of clinical features between community-associated and healthcare-associated methicillin-resistant Staphylococcus aureus keratitis. J Ophthalmol 2015:923941. doi:10.1155/2015 1923941

36. Shetty R, Kaweri L, Nuijts RM, Nagaraja H, Arora V, Kumar RS (2014) Profile of microbial keratitis after corneal collagen crosslinking. Biomed Res Int 2014:340509. doi:10.1155/2014/340509

37. Sueke H, Shankar J, Neal T, Winstanley C, Tuft S, Coates R, Horsburgh MJ, Kaye S (2013) lukSF-PV in Staphylococcus aureus keratitis isolates and association with clinical outcome. Invest Ophthalmol Vis Sci 54:3410-3416. doi:10.1167/iovs.12-11276

38. Zhu H, Conibear TC, Bandara R, Aliwarga Y, Stapleton F, Willcox MD (2006) Type III secretion system-associated toxins, proteases, serotypes, and antibiotic resistance of Pseudomonas aeruginosa isolates associated with keratitis. Curr Eye Res 31:297-306. doi: $10.1080 / 02713680500536746$

39. Fleiszig SM, Wiener-Kronish JP, Miyazaki H, Vallas V, Mostov KE, Kanada D, Sawa T, Yen TS, Frank DW (1997) Pseudomonas aeruginosa-mediated cytotoxicity and invasion correlate with distinct genotypes at the loci encoding exoenzyme S. Infect Immun 65: 579-586

40. Lomholt JA, Poulsen K, Kilian M (2001) Epidemic population structure of Pseudomonas aeruginosa: evidence for a clone that is pathogenic to the eye and that has a distinct combination of virulence factors. Infect Immun 69:6284-6295. doi:10.1128 /iai.69.10.6284-6295.2001

41. Thibodeaux BA, Caballero AR, Marquart ME, Tommassen J, O'Callaghan RJ (2007) Corneal virulence of Pseudomonas aeruginosa elastase B and alkaline protease produced by Pseudomonas putida. Curr Eye Res 32:373-386. doi:10.1080 /02713680701244181

42. Zaidi TS, Zaidi T, Pier GB, Priebe GP (2012) Topical neutralization of interleukin-17 during experimental Pseudomonas aeruginosa corneal infection promotes bacterial clearance and reduces pathology. Infect Immun 80:3706-3712. doi:10.1128/iai.00249-12

43. Tajima K, Miyake T, Koike N, Hattori T, Kumakura S, Yamaguchi T, Matsumoto T, Fujita K, Kuroda M, Ito N, Goto H (2014) In vivo challenging of polymyxins and levofloxacin eye drop against multidrug-resistant Pseudomonas aeruginosa keratitis. J Infect Chemother 20:343-349. doi:10.1016/j.jiac.2013.10.015

44. Goldblum D, Frueh BE, Sarra GM, Katsoulis K, Zimmerli S (2005) Topical caspofungin for treatment of keratitis caused by Candida albicans in a rabbit model. Antimicrob Agents Chemother 49: 1359-1363. doi:10.1128/aac.49.4.1359-1363.2005

45. Jackson BE, Mitchell BM, Wilhelmus KR (2007) Corneal virulence of Candida albicans strains deficient in Tup1-regulated genes. Invest Ophthalmol Vis Sci 48:2535-2539. doi:10.1167/iovs.060909

46. Yuan X, Hua X, Wilhelmus KR (2010) The corneal expression of antimicrobial peptides during experimental fungal keratitis. Curr Eye Res 35:872-879. doi:10.3109/02713683.2010.495812

47. Yavas GF, Ozturk F, Kusbeci T, Cetinkaya Z, Ermis SS, Kiraz N, Inan UU (2008) Antifungal efficacy of voriconazole, itraconazole and amphotericin b in experimental Fusarium solani keratitis. Graefes Arch Clin Exp Ophthalmol 246:275-279. doi:10.1007 /s00417-007-0687-1

48. Das S, Sharma S, Mahapatra S, Sahu SK (2015) Fusarium keratitis at a tertiary eye care centre in India. Int Ophthalmol 35:387-393. doi:10.1007/s10792-014-9961-5

49. Ledbetter EC, Norman ML, Starr JK (2015) In vivo confocal microscopy for the detection of canine fungal keratitis and monitoring of therapeutic response. Vet Ophthalmol. doi:10.1111/vop.12287

50. Thomas PA, Kaliamurthy J (2013) Mycotic keratitis: epidemiology, diagnosis and management. Clin Microbiol Infect 19:210-220. doi:10.1111/1469-0691.12126

51. Zhu JL, Gao XR, Cui HP, Lang LL, Li Q, Liao X (2011) Experimental model of Fusarium solani keratitis in rats. Int $\mathrm{J}$ Ophthalmol 4:371-376. doi:10.3980/j.issn.2222-3959.2011.04.09

52. Pan X, Wang Y, Zhou Q, Chen P, Xu Y, Chen H, Xie L (2011) Activation of focal adhesion kinase enhances the adhesion of Fusarium solani to human corneal epithelial cells via the tyrosinespecific protein kinase signaling pathway. Mol Vis 17:638-646

53. Ojeda JL, Ventosa JA, Piedra S (2001) The three-dimensional microanatomy of the rabbit and human cornea. A chemical and mechanical microdissection-SEM approach. J Anat 199:567-576

54. Marquart ME (2011) Animal models of bacterial keratitis. J Biomed Biotechnol 2011:680642. doi:10.1155/2011/680642

55. Durr UH, Sudheendra US, Ramamoorthy A (2006) LL-37, the only human member of the cathelicidin family of antimicrobial peptides. Biochim Biophys Acta 1758:1408-1425. doi:10.1016/j. bbamem.2006.03.030

56. Royle L, Matthews E, Corfield A, Berry M, Rudd PM, Dwek RA, Carrington SD (2008) Glycan structures of ocular surface mucins in man, rabbit and dog display species differences. Glycoconj J 25: 763-773. doi:10.1007/s10719-008-9136-6

57. Sriram S, Gibson DJ, Robinson P, Pi L, Tuli S, Lewin AS, Schultz $G$ (2014) Assessment of anti-scarring therapies in ex vivo organ cultured rabbit corneas. Exp Eye Res 125:173-182. doi:10.1016/j. exer.2014.06.014

58. Marino A, Pergolizzi S, Lauriano ER, Santoro G, Spataro F, Cimino F, Speciale A, Nostro A, Bisignano G (2015) TLR2 activation in corneal stromal cells by Staphylococcus aureus-induced keratitis. Acta Pathol Microbiol Immunol Scand 123:163-168. doi:10.1111 /apm. 12333

59. Posch LC, Zhu M, Robertson DM (2014) Multipurpose care solution-induced corneal surface disruption and Pseudomonas aeruginosa internalization in the rabbit corneal epithelium. Invest Ophthalmol Vis Sci 55:4229-4237. doi:10.1167/iovs.14-14513 